\title{
Impacto de cenários futuros de clima no zoneamento agroclimático do trigo na região Sul do Brasil
}

\author{
Anderson Santi ${ }^{1(*)}$, Matheus Boni Vicari ${ }^{2}$, Cristina Pandolfo ${ }^{3}$, Genei Antonio Dalmago ${ }^{1}$, Angelo Mendes Massignam ${ }^{3}$ e Aldemir \\ Pasinato $^{1}$ \\ ${ }^{1}$ Embrapa Trigo, Rodovia BR 285, km 294, Caixa Postal 3081, CEP 99050-970 Passo Fundo, RS. E-mail: anderson.santi@embrapa.br, \\ genei.dalmago@embrapa.br e aldemir.pasinato@embrapa.br \\ ${ }^{2}$ University College London, Gower St, Bloomsbury, London WC1E 6BT, E-mail: matheus.boni.vicari@gmail.com \\ ${ }^{3}$ Empresa de Pesquisa Agropecuária e Extensão Rural de Santa Catarina - Epagri/CIRAM, Rodovia Admara Gonzaga, 1347, Itacorubi - Caixa Postal 502, \\ CEP 88034-901 Florianópolis, SC. E-mails: cristina@epagri.sc.gov.br e massigna@epagri.sc.gov.br \\ ${ }^{(*)}$ Autor para correspondência
}

\section{INFORMAÇÕES}

História do artigo:

Recebido em 10 de Julho de 2017

Aceito em 27 de Dezembro de 2017

Termos para indexação:

temperatura do ar

equações topotérmicas

período de semeadura

região de aptidão
RESUMO

As mudanças climáticas ampliam o risco sobre a oferta global de alimentos por afetarem significativamente as condições de crescimento e desenvolvimento das plantas. O trigo tem papel preponderante na alimentação humana e animal e é altamente responsivo aos elementos climáticos, sendo então vulnerável à mudança de clima. 0 objetivo deste trabalho foi avaliar o impacto de cenários futuros de clima no zoneamento agroclimático da cultura do trigo na região Sul do Brasil. 0 estudo considerou dois cenários climáticos futuros estabelecidos pelo Painel Intergovernamental sobre Mudança do Clima, centrados no período 2071 2100: o A2, ou mais pessimista, e o B2, ou mais otimista, que foram comparados ao cenário Atual (1961 - 1990). o período de cultivo do trigo indicado pelo zoneamento agroclimático será reduzido em toda a região Sul do País, com a temperatura sendo a principal responsável pela limitação do número de decêndios aptos à semeadura. A cultura do trigo terá sua área de semeadura potencial reduzida principalmente no Paraná, em ambos cenários, e no oeste do Rio Grande do Sul somente no pior cenário.

\section{Introdução}

No âmbito das principais culturas agrícolas destinadas à produção de alimentos, o trigo responde por aproximadamente $21 \%$ da demanda mundial. Para atender à necessidade crescente dos países em desenvolvimento, deverá ocorrer aumento na produção deste cereal em 1,6\% ao ano até 2020 , ou 2,6\% ao ano se considerado o consumo animal, fato que resulta no necessário incremento de 2,6 toneladas para 3,5 toneladas de grãos por hectare durante os próximos 25 anos (ORTIZ et al., 2008). Em escala global, tais projeções são corroboradas por Fresco (2009), que sugere 
o dobro da produtividade atual para os cerais até 2030, bem como pela FAO (Food and Agricultural Organization), que embora numa perspectiva mais amena, entretanto, não menos preocupante, estima o necessário aumento de produtividade para o trigo em $70 \%$ até 2050 (LIPPER et al., 2010). No entanto, Ray et al. (2013) ao avaliarem globalmente mais de 2,5 milhões estatísticas agrícolas, estimaram taxa de aumento no rendimento de trigo de $0,9 \%$ ao ano, está muito aquém da necessária ( $2,4 \%$ ao ano) para dobrar a produção mundial em 2050 e, além disso, demonstram que a essa taxa atual seria possível alcançar apenas $38 \%$ da meta necessária para o trigo.

No Brasil, a área colhida de trigo foi de 2,1 milhões de ha em 2016, com variação entre 1,9 a 2,8 milhões de hectares nos últimos cinco anos (IBGE, 2017a, b). Geralmente, a tomada de decisão para semeadura da cultura está associada a fatores como condições meteorológicas correntes, preços pagos ao produto, preço mínimo e garantia de seguro (que são dependentes da política governamental). A produção média de trigo no período 2012-2016 foi de 5,7 milhões de toneladas, suficiente para suprir aproximadamente $50 \%$ da necessidade interna do cereal que varia entre 9 e 11 milhões de toneladas (IBGE, 2017a, b). No período acima, a região Sul do país foi responsável por 92\% do total produzido, sendo que os estados do Paraná, Santa Catarina e Rio Grande do Sul responderam por $49 \%$, $4 \%$, e $39 \%$ da produção nacional de trigo, respectivamente (IBGE, 2017a, b).

0 trigo tem resposta pronunciada à variação dos elementos climáticos, como geada (SCHEEREN et al., 2000) e/ ou seca no espigamento, umidade relativa do ar e/ou temperaturas elevadas na fase de enchimento de grãos e chuva na colheita (CUNHA et al., 2001). Portanto, a demarcação dos limites das regiões homogêneas de adaptação de cultivares de trigo no Brasil considera a precipitação pluvial na estação de crescimento da cultura, a quantidade de frio invernal, o excesso de calor no enchimento de grãos, a altitude e a série histórica de rendimento de grãos (CUNHA et al., 2006). Além disso, também é considerado o regime hídrico durante a estação de crescimento do trigo, que define, em macroescala, duas regiões: uma úmida, em que não há estação seca e a precipitação pluvial supera a evapotranspiração, e outra seca, onde normalmente ocorrem excesso de calor e déficit hídrico (CUNHA et al., 2011).

$O$ aumento na frequência de extremos climáticos, bem como a elevação da temperatura global (SOLOMON et al., 2009) em até $3,7^{\circ} \mathrm{C}$ no cenário de mudança climática (IPCC, 2014), são associados ao incremento da concentração de gases de efeito estufa, especialmente de $\mathrm{CO}_{2}$, de $\mathrm{CH}_{4}$ e de $\mathrm{N}_{2} \mathrm{O}$, apontados como causadores do aquecimento global (TIAN et al., 2016), que tiveram acréscimos respectivos em $40 \%, 150 \%$ e $20 \%$ desde o início da revolução industrial em 1750 (CIAIS et al., 2013). No âmbito dos gases de efeito estu- fa, o $\mathrm{CO} 2$ responde por até $75 \%$ do total emitido, e embora as taxas de sequestro no solo e nos oceanos tenham aumentado de 1959 a 2010, ainda ocorre acúmulo anual significativo na atmosfera, da ordem de 15 Pg (1 Petagrama= 1 Giga tonelada $=1$ bilhão de toneladas), no período $2000 \mathrm{a}$ 2010 (BALLANTYNE et al., 2012).

O panorama apresentado preocupa ao se considerar o potencial impacto destas mudanças sobre as culturas. Para a cultura do trigo, os estudos indicam que o aumento da concentração de $\mathrm{CO}_{2}$ no ambiente teria efeitos diferenciados sobre a cultura, sendo beneficiada por apresentar metabolismo $C_{3}$ (TUBIELLO et al., 2000), com aumento do rendimento (WALL et al., 2006; ASSENG et al., 2004), pois ocorreria acréscimo na matéria prima para a realização de fotossíntese pelas plantas (MAGRIN et al., 2009). Porém, haveria aumento da incidência de ferrugem no trigo (CHAKRABORTY et al., 2011) e redução na qualidade do grão (HÖGY et al., 2009; BLOOM et al., 2014), em especial no teor de proteína do grão e da farinha (ZISKA et al., 2004) e na concentração de micronutrientes, como zinco e ferro (MYERS et al., 2014).

A elevação da temperatura global interfere no ciclo das plantas, com reflexos diretos e nem sempre positivos sobre sua fenologia (BUITENWERF et al., 2015). Dentre os elementos meteorológicos que interferem no desenvolvimento da cultura do trigo, estão a temperatura e o fotoperíodo (RIBEIRO et al., 2009). O fato de o trigo necessitar de exposição a horas de frio não congelante para que ocorra indução ao florescimento (PAULSEN, 1987) e, principalmente, ter sua maior vulnerabilidade a altas temperaturas durante o período reprodutivo, em especial no espigamento (MARCELLOS; SINGLE, 1972; MOTA, 1989), impõe à cultura riscos em cenários de mudança de clima, pelo aumento de temperatura esperado (MARENGO et al., 2007; TRAVASSO et al., 2008; SOLOMON et al., 2009) e observado (MARENGO; CAMARGO, 2008) para a região do estudo. Em escala global, estudos com trigo demonstram impactos fenológicos, como encurtamento do ciclo da cultura (ZHENG et al., 2016) e cenários que implicam em redução no rendimento (LOBELL et al., 2008), com variação média de 5,7\% (LIU et al., 2016) a 6\% por grau de acréscimo da temperatura global (ASSENG et al., 2015).

Portanto, estudar o desempenho da cultura do trigo, considerando os cenários de mudança de clima, tem grande relevância econômica e social para o setor ao qual a cultura é vinculada, pois, além de apresentar panorama acerca das regiões que possuem maior vulnerabilidade para o trigo, fornece subsídios para tomada de decisão acerca de medidas de adaptação passíveis de serem implementadas visando a diminuir os impactos. A dimensão do problema ou do benefício gerado à atividade tritícola estará associada à intensidade experimentada da mudança climática e à capacidade de adaptação (natural ou suportada por ações 
humanas) nas diversas regiões de produção de trigo.

Estudos complementares, em escala regionalizada, são imprescindíveis para auxiliar na tomada de decisão relacionada a ações de adaptação e de mitigação dos efeitos das mudanças climáticas sobre a cultura do trigo. Dessa forma, o objetivo desse trabalho foi avaliar o impacto de cenários de clima futuros no zoneamento agroclimático da cultura do trigo na região Sul do Brasil.

\section{Material e Métodos}

O estudo foi desenvolvido no âmbito da região Sul do Brasil, englobando os estados do Paraná (399 municípios), Santa Catarina (293 municípios) e Rio Grande do Sul (496 municípios).

Para avaliar o impacto das mudanças climáticas no zoneamento agroclimático do trigo na região Sul do Brasil foram utilizados três cenários: cenário Atual (1961-1990) e cenários futuros A2 e B2 (2071-2100). 0 cenário A2 (pessimista), prevê aumento de temperatura entre $2,0{ }^{\circ} \mathrm{C}$ e $5,4^{\circ} \mathrm{C}$ até 2100 e o B2 (otimista), estima aumento de temperatura entre $1,4^{\circ} \mathrm{C}$ e $3,8{ }^{\circ} \mathrm{C}$ até 2100 (CUBASCH et al., 2001). Os cenários A2 e B2 foram utilizados para estimar os padrões climáticos futuros de temperatura do ar e de precipitação, considerando os dados médios observados no período de 1961 - 1990.

Inicialmente, foram extraídos dos mapas de relevo da região Sul (MIRANDA, 2005) os pontos de altitude georreferenciados em malha regular de $900 \mathrm{~m}$, procedendo-se à retirada de ruídos, como pontos sem informação com dados zerados. Essas informações foram utilizadas para a estimativa das equações topotérmicas.

Os dados diários de precipitação e de temperaturas máxima e mínima foram obtidos do modelo climático regional HadRM3P, detalhado em Jones et al. (2004), para estabelecer a base climática (1961-1990) e os cenários futuros A2 e B2, do período 2071 - 2100. Este modelo, desenvolvido pelo Hadley Centre, faz parte do PRECIS (Providing Regional Climates for Impacts Studies) e integra a resolução de 19 níveis na vertical (até $30 \mathrm{~km}$ na estratosfera) e $50 \mathrm{~km}$ na horizontal ( 0,5 x 0,5 grau), além de quatro níveis no solo (MARENGO et al., 2009).

Os dados da base climática gerada pelo modelo HadRM3P foram verificados quanto à capacidade de representar o clima presente e sua variabilidade em uma escala menor (região Sul), tanto para as temperaturas mínimas e máximas (Camargo et al., 2012) quanto para a precipitação (CAMARGO et al., 2011). Para tanto, os autores utilizaram estações meteorológicas de cada um dos três Estados (12 estações no RS, 10 estações em SC e 9 estações no PR) que possuíam o período de dados coincidente com a base climática gerada pelo modelo, ou seja, 1961-1990.

Após todos os dados climáticos gerados pelo modelo em escala $0,5 \times 0,5$ grau $(\sim 50 \mathrm{~km} \times 50 \mathrm{~km})$ foram interpolados por krigagem ordinária e extraídos para a malha regular de $900 \mathrm{~m}$ com o software ArcGis 10.

Para delimitação das regiões de aptidão da cultura do trigo, utilizaram-se índices descritos em Cunha et al. (2001), bem como ajustes realizados pelo grupo de pesquisa, visando padronizar as informações de modo a atender as necessidades do software de zoneamento utilizado. Para o zoneamento agroclimático da cultura do trigo, foi utilizado como ferramenta o sistema especialista Zonexpert 1.0 (PANDOLFO et al., 1999), software padrão de trabalhos de zoneamento no estado de Santa Catarina. Neste sistema, foi inserida a base de dados climática, sendo adicionados os seguintes critérios para fins de zoneamento agroclimático do trigo: ciclo da cultura - médio (140 dias); temperatura média mensal menor que $20^{\circ} \mathrm{C}$ no terceiro, quarto e quinto mês após a semeadura; temperatura mínima mensal maior ou igual a $9{ }^{\circ} \mathrm{C}$ em todos os meses do ciclo; temperatura média mensal menor que $23^{\circ} \mathrm{C}$ na semeadura; e índice de satisfação da necessidade de água (ISNA) maior ou igual a 0,60 para todos os decêndios do ciclo. Dessa forma, indicou-se a semeadura quando mais de $20 \%$ da área do município estudado apresentou-se apta para tal, no decêndio considerado.

Foi utilizado o balanço hídrico climatológico desenvolvido por Thornthwaite \& Mather (1955), com CAD de 100 $\mathrm{mm}$ e dados de precipitação para os cenários Atual, A2 e B2. Para o cálculo da evapotranspiração de referência (ETo), foram utilizados dados de temperatura média provenientes do PRECIS para os mesmos cenários. A ETo foi estimada pelo método de Thornthwaite como descrito em Bergamaschi et al. (1999).

Com base nos dados observados para a região Sul de 1961 a 2008, foram desenvolvidas equações topotérmicas visando à obtenção das temperaturas máximas, médias e mínimas mensais do ar (variáveis dependentes) e as de risco climático, que foram interpoladas na malha de $900 \mathrm{~m}$ para os 36 decêndios do ano. Os dados de precipitação do período avaliado foram espacializados por krigagem ordinária e extraídas também para a malha de $900 \mathrm{~m}$. Os riscos de temperaturas mínimas avaliavam temperaturas menores que $5{ }^{\circ} \mathrm{C}, 4{ }^{\circ} \mathrm{C}, 3{ }^{\circ} \mathrm{C}, 2^{\circ} \mathrm{C}, 1^{\circ} \mathrm{C}, 0{ }^{\circ} \mathrm{C}$ e $-1{ }^{\circ} \mathrm{C}$. Considerando que a temperatura ótima para o trigo varia de $18-24{ }^{\circ} \mathrm{C}$ e temperaturas superiores a esta faixa, especialmente acima de $30{ }^{\circ} \mathrm{C}$ implica em perdas de qualidade e rendimento de grãos (STONE; NICOLAS, 1994), avaliou-se para os riscos de temperaturas máximas aquelas maiores que $29^{\circ} \mathrm{C}, 30^{\circ} \mathrm{C}, 31$ ${ }^{\circ} \mathrm{C}, 32{ }^{\circ} \mathrm{C}, 33^{\circ} \mathrm{C}, 34^{\circ} \mathrm{C}$ e $35^{\circ} \mathrm{C}$.

Para estimar a disponibilidade hídrica, foi utilizado o índice de satisfação da necessidade de água (ISNA), que é definido pela relação existente entre a evapotranspiração real e a evapotranspiração máxima da cultura. Para cruzamento com os dados de cultura e geração dos cenários, 
Figura 1. Municípios indicados para cultivo de trigo na região sul do Brasil para os cenários climáticos: a) Atual (1961-1990), b) B2 e, c) A2. Passo Fundo, RS, 2017.

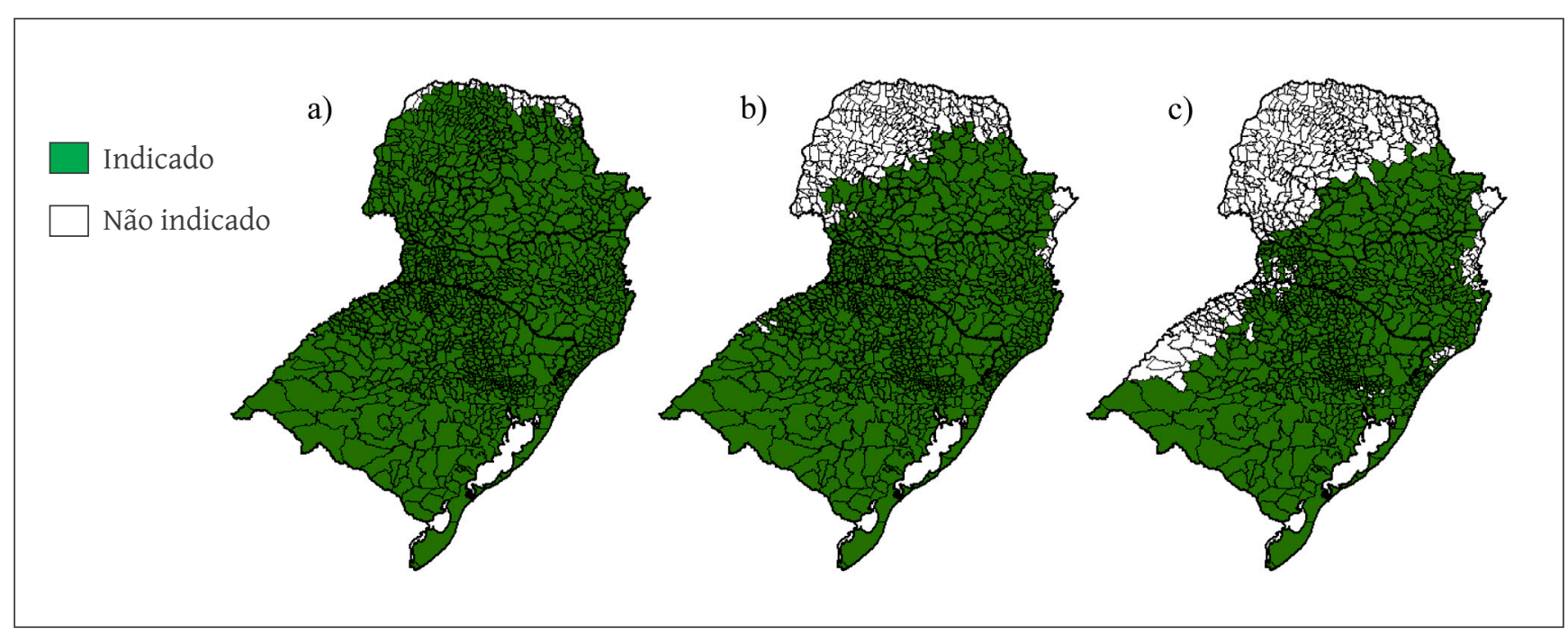

foram utilizados valores médios mensais de ISNA. Para o zoneamento agroclimático não foram utilizados critérios edáficos.

Com auxílio do software ArcGis 10, calculou-se o aumento ou a retração das áreas e/ou modificações no período de semeadura do trigo (número de decêndios aptos à semeadura), comparando-se os resultados observados entre os cenários agrícolas Atual, A2 e B2. Maiores detalhes sobre a metodologia utilizada neste trabalho podem ser consultados em Massignam et al. (2015).

\section{Resultados e discussão}

Considerando somente os critérios de zoneamento utilizados nesse trabalho para a cultura do trigo, observou-se que, para o cenário Atual, há potencial de cultivo de trigo em praticamente toda a região Sul (Figura 1). No cenário A1, a região oeste do Rio Grande do Sul e a região mais quente do Paraná apresentaram limitação ao cultivo do trigo, pois o aumento de temperatura do ar, esperado em função de ser apontado por outros autores (MARENGO et al., 2010) ficou acima do limite crítico durante o ciclo da cultura. Tal fato ocorreu também no cenário B2, com predominância no estado do Paraná.

Tanto no cenário otimista B2 quanto no pessimista A2, houve redução da quantidade de municípios aptos para cultivo, principalmente na região noroeste do Rio Grande do Sul e no norte do estado do Paraná (Figura 1). A escala de redução foi menor no cenário B2, fato provavelmente associado à menor repercussão desse cenário nos parâmetros de temperatura e precipitação, que são fatores limitantes ao crescimento e desenvolvimento da cultura (CUNHA et al., 2011).

A Figura 2 apresenta informações em relação aos períodos aptos para semeadura do trigo. Houve redução no número de decêndio indicados para semeadura, principalmente no Paraná e no oeste do Rio Grande do Sul, o que pode ser devido a alterações positivas nas temperaturas do ar. Esse resultado é preocupante, pois temperaturas elevadas podem causar estresse térmico (FAROOQ et al., 2011), reduzindo a capacidade metabólica das plantas, resultando em perdas no rendimento de grãos de trigo, como já predito por Asseng et al. (2015). Em estudo realizado por Streck e Alberto (2006) para as condições de Santa Maria, RS, os autores concluíram que mudança climática com temperaturas acima de $3{ }^{\circ} \mathrm{C}$ terá efeito deletério sobre o rendimento de trigo, anulando, até mesmo, efeitos benéficos advindos do aumento da concentração de gás carbônico atmosférico.

O período apto de semeadura do trigo sofreu redução entre 5 a 15 decêndios, tanto no cenário A2 quanto no B2, nos três estados do Sul (Figura 3). Principalmente no Rio Grande do Sul, no cenário A1, mais de 300 municípios apresentaram perda entre 5 e 10 decêndios, encurtando o período de semeadura da cultura.

No cenário B2, os três estados tiveram redução de 5 a 10 decêndios indicados ao cultivo do trigo em mais de 200, 150 e 330 municípios, respectivamente, para o Paraná, Santa Catarina e no Rio Grande do Sul (Figura 3).

Considerando a área porcentual dos estados sob influência da mudança climática, observa-se que, em praticamente $100 \%$ da área dos estados do Sul, houve alteração negativa no número de decêndios recomendados para a cultura do trigo, tanto no cenário $\mathrm{B} 2$, quanto no cenário $\mathrm{A} 1$ (Figura 4), implicando, portanto, na diminuição do período indicado de semeadura de trigo. Independente do cenário avaliado, este fato é preocupante, pois dificulta ao agricultor o estabelecimento da cultura, principalmente na ocorrência de condições climáticas adversas, por reduzir a janela apta à semeadura do trigo. 
Figura 2. Mudanças projetadas para o número de decêndios indicados para semeadura de trigo para os cenários B2 e A2 em relação ao Atual. Passo Fundo, RS, 2017.

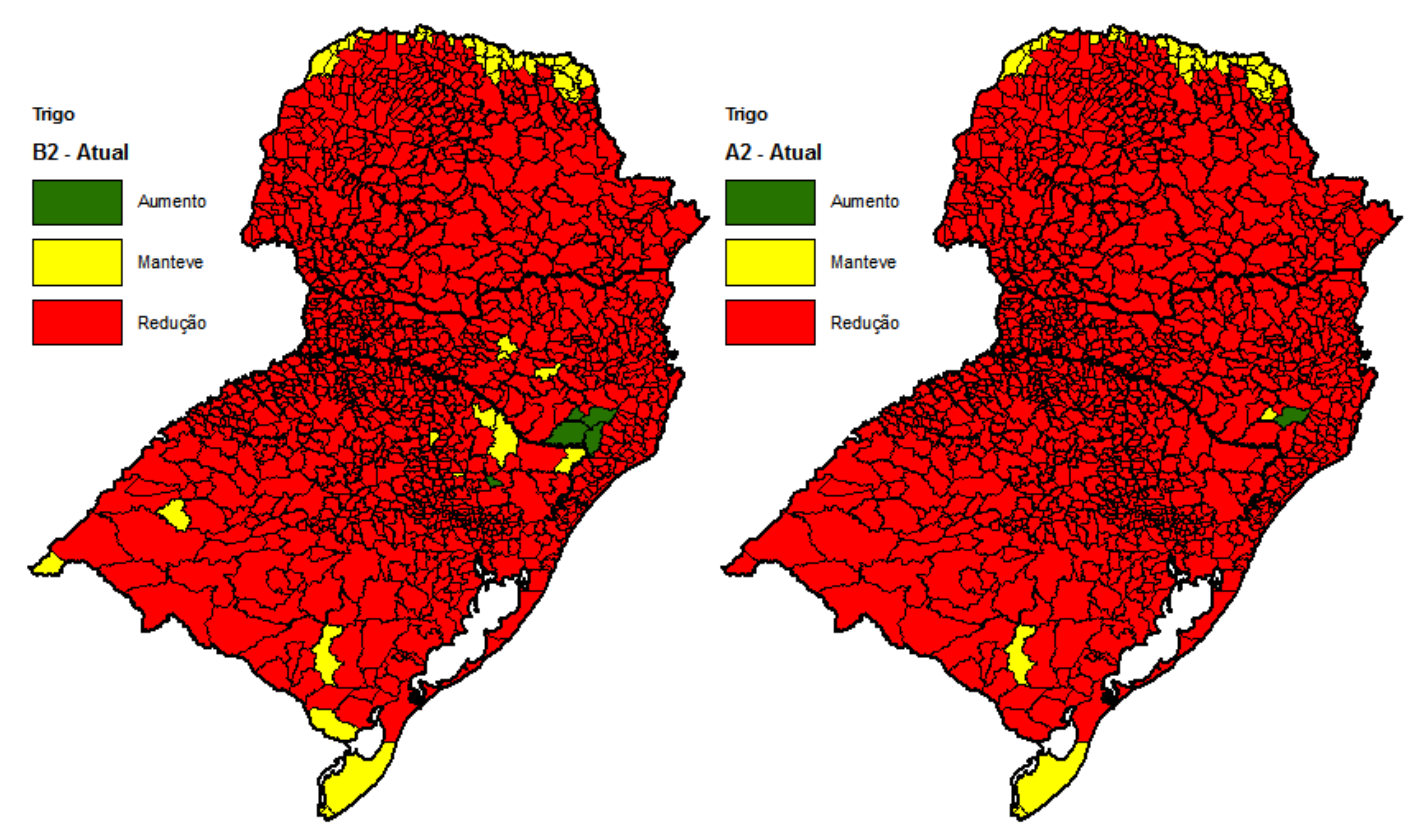

Figura 3. Mudanças no número de decêndios com semeadura indicada para trigo nos municípios do Paraná (PR), Santa Catarina (SC) e Rio Grande do Sul (RS), para os cenários A2 e B2. Passo Fundo, RS, 2017.
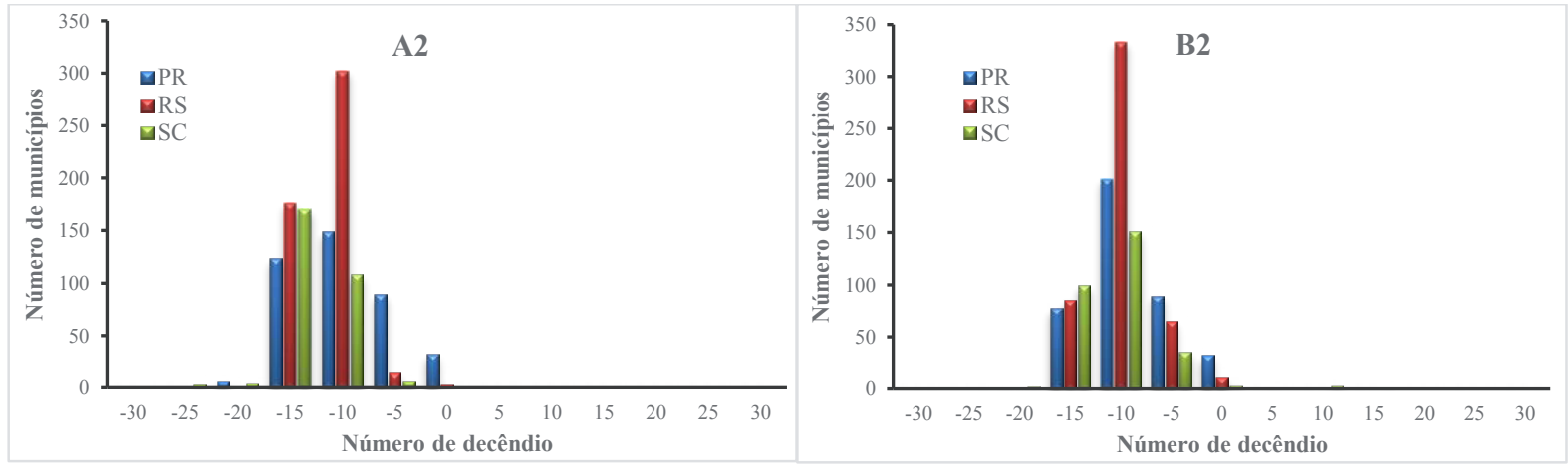

Figura 4. Porcentagem da área municipal total acumulada em função da variação, em número de decêndios, do período de semeadura da cultura de trigo em municípios do Paraná (PR), Santa Catarina (SC) e Rio Grande do Sul (RS), para os cenários A2 e B2. Passo Fundo, RS, 2017.
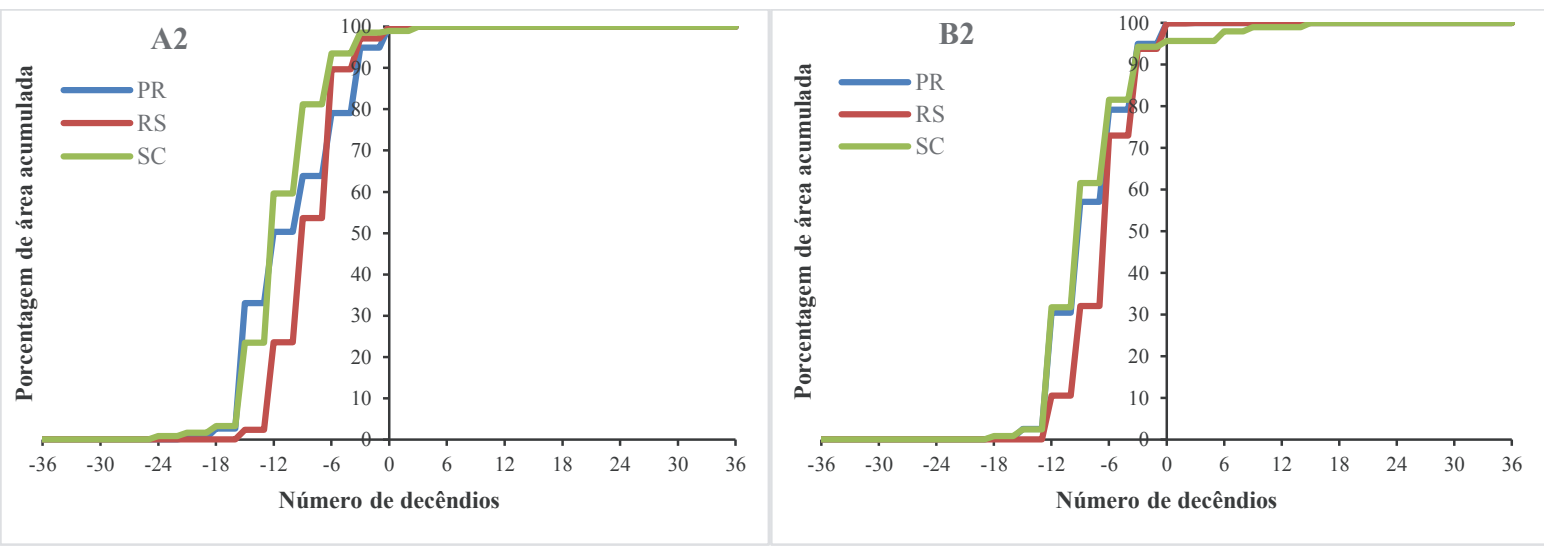
Tabela 1. Estimativa de áreas, nos cenários Atual e futuros, atuais e futuras indicadas para cultivo de trigo e variações de áreas decorrentes dos cenários A2 e B2, por estado e total da região Sul do Brasil. Passo Fundo, RS, 2017.

\begin{tabular}{lcccc} 
Cenários & \multicolumn{3}{c}{ Estado/área $\left(\mathbf{k m}^{2}\right)$} \\
\hline Atual & Paraná & Santa Catarina & Rio Grande do Sul & Região Sul \\
\hline A2 & 191.213 & 96.001 & 273.035 & 560.248 \\
\hline B2 & 77.349 & 86.407 & 242.405 & 406.161 \\
\hline A2 & 112.446 & 93.874 & 272.076 & 478.395 \\
\hline B2 & $-59,5$ & Variação relativa ao cenário Atual (\%) \\
\hline
\end{tabular}

Grande parte da área foi afetada quando houve redução entre 6 e 12 decêndios, alcançando média aproximada de $60 \%$ na região Sul, no pior cenário (A2). No cenário B2, o maior porcentual de área com redução decendial ocorreu quando houve diminuição de até 6 decêndios, abrangendo, em média, 50\% da área região Sul (Figura 4).

Pela análise da Tabela 1, cujas informações foram estimadas com base nos cenários estudados, observa-se que a área indicada para cultivo de trigo no Paraná e no Rio Grande do Sul sofrerá redução de 11,39 (59,5\%) e 0,95 $(11,2 \%)$ milhões de hectares no cenário A2. Para o cenário B2, o Paraná deverá ser o estado mais prejudicado da região Sul, com perda de 7,88 milhões de hectares $(41,2 \%)$ aptos para cultivo.

De maneira geral, a região Sul terá uma redução potencial de 15,4 (27,5\%) milhões de hectares no cenário A2, e de 8,2 (14,6\%) milhões de hectares no cenário B2 (Tabela 1).

\section{Conclusões}

Na região Sul do Brasil, a cultura do trigo terá sua área de semeadura potencial reduzida, principalmente no $\mathrm{Pa}$ raná, em ambos cenários de clima estudados (pessimista e otimista de mudança climática), e no oeste do Rio Grande do Sul, somente no cenário A2.

o período de cultivo do trigo indicado pelo zoneamento agroclimático será reduzido em toda a região Sul do País, com a temperatura sendo a principal responsável pela limitação do número de decêndios aptos à semeadura.

\section{Agradecimentos}

Os autores agradecem à Finep, pelo apoio financeiro, ao CNPq, pela concessão de bolsas, e à Embrapa, à Fundação Universidade Regional de Blumenau e à Epagri, pelo apoio institucional. Também agradecem ao INPE pela disponibilização dos modelos meteorológicos.

\section{Referências bibliográficas}

ASSENG, S.; EWERT, F.; MARTRE, P.; RÖTTER, R. P.; LOBELL, D. B.; CAMMARANO, D.; KIMBALL, B. A.; OTTMAN, M. J.; WALL, G.W.; WHITE, J.W.; REYNOLDS, M. P.; ALDERMAN, P. D.; PRASAD, P. V. V.; AGGARWAL, P. K.; ANOTHAI, J.; BASSO, B.; BIERNATH, C.; CHALLINOR, A. J.; SANCTIS, G. DE; DOLTRA, J.; FERERES, E.; GARCIA-VILA, M.; GAYLER, S.; HOOGENBOOM, G.; HUNT, L. A.; IZAURRALDE, R. C.; JABLOUN, M.; JONES, C. D.; KERSEBAUM, K. C.; KOEHLER, A-K.; MÜLLER, C.; NARESH KUMAR, S.; NENDEL, C.; O'LEARY, G.; OLESEN, J. E.; PALOSUO, T.; PRIESACK, E.; EYSHI REZAEI, E.; RUANE, A. C.; SEMENOV, M. A.; SHCHERBAK, I.; STÖCKLE, C.; STRATONOVITCH, P.; STRECK, T.; SUPIT, I.; TAO, F.; THORBURN, P. J.; WAHA, K.; WANG, E.; WALLACH, D.; WOLF, J.; ZHAO, Z.; ZHU, Y. Rising temperatures reduce global wheat production. Nature Climate Change, v. 5, p. 143 - 147, 2015. DOI: 10.1038/nclimate2470

ASSENG, S.; JAMIESON, P.D.; KIMBALL, B.; PINTER, P.; SAYRE, K.; BOWDEN, J.W.; HOWDEN, S.M. Simulated wheat growth affected by rising temperature, increased water deficit and elevated atmospheric $\mathrm{CO}_{2}$. Field Crops Research, v. 85, p. 85 - 102, 2004.

BALLANTYNE, A. P.; ALDEN, C. B.; MILLER, J. B.; TANS, P. P.; WHITE, J. W. C. Increase in observed net carbon dioxide uptake by land and oceans during the past 50 years. Nature, v. 488, p.70-72, 2012.

BERGAMASCHI, H.; BERLATO, M. A.; MATZENAUER, R.; FONTANA, D. C.; CUNHA, G. R. da; SANTOS, M. L. V.; FARIAS, J. R. B.; BARNI, N. A. Agrometeorologia aplicada à irrigação. 2 ed. Porto Alegre: Editora da Universidade/UFRGS, 1999. 125p.BLOOM, A. J.; BURGER, M.; KIMBALL, B. A.; PINTER Jr., P. J. Nitrate assimilation is inhibited by elevated $\mathrm{CO} 2$ in field-grown wheat. Nature Climate Change, v. 4, p. 477 - 480, 2014.

BUITENWERF, R.; ROSEL, L.; HIGGINS, S. I. Three decades of multidimensional change in global leaf phenology. Nature climate Change, v. 5, p. $364-368,2015$.

CAMARGO, C. G. C.; MALANDRIN, D.; MARENGO, J.; BRAGA, H.; PANDOLFO, C.; MASSIGNAM, A. Análises dos padrões climáticos futuros de precipitação na região Sul do Brasil. In: XVII Congresso Brasileiro de Agrometeorologia, 2011, Guarapari - ES. Anais.: XVII Congresso Brasileiro de Agrometeorologia, 2011.

CAMARGO, C. G. C.; MARENGO, J. A.; BRAGA, H. J. Variabilidade interanual da temperatura do ar na região Sul do Brasil. In: XVII Congresso Brasileiro de Meteorologia, 2012, Gramado/RS. XVII Congresso Brasileiro de Meteorologia, 2012.

CHAKRABORTY, S.; LUCK, J.; HOLLAWAY; G.; FITZGERALD, G.; WHITE, N. Rust-proofing wheat for a changing climate. Euphytica, v. 179, p. 19 - 32, 2011.

CIAIS, P.; SABINE, C.; BALA, G.; BOPP, L.; BROVKIN, V.; CANADELL, J.; CHHABRA, A.; DEFRIES, R.; GALLOWAY, J.; HEIMANN, M.; JONES, C.; LE QUÉRÉ, C.; MYNENI, R. B.; PIAO; S.; THORNTON, P. Carbon and other biogeochemical cycles, p. 465 - 575, 2013. In: STOCKER, T. F.; QIN, D.; PLATTNER, G. K.; TIGNOR, M.; ALLEN, S.K.; BOSCHUNG, J.; NAUELS, A.; XIA, Y.; BEX, V.; MIDGLEY, P. M. (eds.). IPCC: Climate Change 2013: the Physical Science Basis. Contribution of Working Group I to the Fifth Assessment Report of the Intergovernmental Panel on Climate Change. Cambridge University Press, Cambridge, United Kingdom and New York, NY, USA, 2013. 1535p. 
CUBASCH, U.; MEEHL, G.A.; BOER, G.J.; STOUFFER, R.J.; DIX, M.; NODA, A.; SENIOR, C.A.; RAPER, S.; YAP, K.S. Projections of future climate change. p. 524 - 582, 2001. In: HOUGHTON, J. T.; DING, Y.; GRIGGS, D. J.; NOGUER, M.; VAN DER LINDEN, P. J.; DAI, X.; MASKELL, K.; JOHNSON, C. A. (eds.). IPCC: Climate Change 2001: the Scientific Basis. Contribution of Working Group I to the Third Assessment Report of the Intergovernmental Panel on Climate Change. Cambridge University Press, Cambridge, United Kingdom and New York, NY, USA, 2001. 881p.

CUNHA, G. R.; HAAS, J. C.; MALUF, J. R. T.; CARAMORI, P. H.; ASSAD, E. D.; BRAGA, H. J.; ZULLO Jr., J.; LAZZAROTTO, C.; GONÇALVES, S.; WREGE, M.; BRUNETTA, D.; DOTTO, S. R.; PINTO, H. S.; BRUNINI, O.; THOMÉ, V. M. R.; ZAMPIERI, S. L.; PASINATO, A.; PIMENTEL, M. B. M.; PANDOLFO, C. Zoneamento agrícola e época de semeadura para trigo no Brasil. Revista Brasileira de Agrometeorologia, Passo Fundo, v.9, n.3, (№ Especial: Zoneamento Agrícola), p. 400 - 414, 2001.

CUNHA, G. R. da; SCHEEREN, P. L.; PIRES, J. L. F.; MALUF, J. R. T.; PASINATO, A.; CAIERÃO, E.; SÓ E SILVA, M.; DOTTO, S. R.; CAMPOS, L. A. C.; FELÍCIO, J. C.; CASTRO, R. L. de; MARCHIORO, V.; RIEDE, C. R.; ROSA FILHO, O.; TONON, V. D.; SVOBODA, L. H. Regiões de adaptação para trigo no Brasil. Passo Fundo: Embrapa Trigo, 2006. 10 p. html. (Embrapa Trigo. Circular Técnica Online, 20). Disponível: <http://www.cnpt. embrapa.br/biblio/ci/p_ci20.htm>. Acesso em: 03 Jul. 2014.

CUNHA, G. R. da; PASINATO, A.; PIMENTEL, M. B. M.; HASS, J. C.; MALUF, J. R. T.; PIRES, J. L. F.; DALMAGO, G. A.; SANTI, A. Regiões para trigo no Brasil: ensaios de VCU, zoneamento agrícola e época de semeadura, p. 27 - 40. In.: PIRES, J. L. F.; VARGAS, L; CUNHA, G. R. Trigo no Brasil: bases para produção competitiva e sustentável. Passo Fundo, Embrapa Trigo, 2011. $488 \mathrm{p}$.

FAROOQ, M.; BRAMLEY, H.; PALTA, J. SIDDIQUE, K. H. M. Heat stress in wheat during reproductive and grain-filling phases. Critical Reviews in Plant Sciences, v. 30, p. 491 - 507, 2011.

FRESCO, L. O. Challenges for food system adaptation today and tomorrow. Environmental Science \& Policy, v. 12, p. 378 - 385, 2009

HÖGY, P.; WIESER, H.; KÖHLER, P.; SCHWADORF, K.; BREUER, J.; FRANZARING, J.; MUNTIFERING, R.; FANGMEIER, A. Effects of elevated $\mathrm{CO} 2$ on grain yield and quality of wheat: results from a 3-year free-air $\mathrm{CO}_{2}$ enrichment experiment. Plant Biology, v. 1 (Suppl. 1), p. 60 - 69, 2009.

IBGE. Produção agrícola municipal. 2017a. Disponível em: <https:// sidra.ibge.gov.br/Tabela/1612\#resultado>. Acesso em: 04 abr. 2017.

IBGE. Levantamento sistemático da produção agrícola. $2017 \mathrm{~b}$. Disponível em: <https://sidra.ibge.gov.br/tabela/1618\#resultado>. Acesso em: 04 abr. 2017.

IPCC. Climate change 2013: the physical science basis: summary for policymakers. 2013. 33 p. Contribution of Working Group I to the Fifth Assessment Report. Twelfth Session of Working Group I. Disponível em: <http://www.ipcc.ch/report/ar5/wg1/docs/WGIAR5_SPM_brochure _ en.pdf>. Acesso em: 04 jul. 2016.

IPCC. Climate change 2014: synthesis report. Contribution of Working Groups I, II and III to the Fifth Assessment Report of the Intergovernmental Panel on Climate Change. Geneva, 2014. 151 p. Edited by The Core Writing Team, Rajendra K. Pachauri, Leo Meyer. Disponível em: <http://ipcc.ch/pdf/assessment-report/ar5/syr/SYR_AR5_FINAL_ full_wcover.pdf>. Acesso em: 25 out. 2017.

JONES, R. G.; NOGUER, M.; HASSELL, D. C.; HUDSON, D.; WILSON, S. S.; JENKINS, G. J.; MITCHELL, J. F. B. Generating high resolution climate change scenarios using PRECIS. Met Office Hadley Centre, Exeter, Uk, April, 2004, 40p.

LIPPER, L.; MANN, W.; MEYBECK, A.; SESSA, R. "Climate-smart" agriculture: policies, practices and financing for food security, adaptation and mitigation. Rome: FAO, 2010. 41 p. Disponível em: <http://www.fao.org/docrep/013/i1881e/i1881e00.pdf>. Acesso em: 7 jul. 2014.
LIU, B.; ASSENG, S.; MÜLLER, C.; EWERT, F.; ELLIOTT, J.; LOBELL, D.B.; MARTRE, P.; RUANE, A. C.; WALLACH, D. JONES, W. J.; ROSENZWEIG, C.; AGGARWARL, P. K; ALDERMAN, P. D.; ANOTHAI, J.; BASSO, B.; BIERNATH, C.; CAMMARANO, D.; CHALLINOR, A.; DERYNG, D.; DE SANCTIS, G.; DOLTRA, J.; FERERES, E.; FOLBERTH, C.; GARCIA-VILA, M.; GAYLER, S.; HOOGENBOOM, G.; HUNT, L. A.; IZAURRALDE, R., C.; JABLOUN, M.; JONES, C. D.; KERSEBAUM, K. C.; KIMBALL, B. A.; KOEHLER, A.K.; KUMAR, S. N.; NENDEL, C.; O'LEARY, G. J.; OLESEN, J. E.; OTTMAN, M. J.; PALOSUO, T.; PRASAD, P. V. V.; PRIESACK, E.; PUGH, T. A. M.; REYNOLDS, M.; REZAEI, E.E.; RÖTTER, R. P.; SCHMID, E.; SEMENOV, M. A.; SHCHERBAK, I.; STEHFEST, E.; STÖCKLE, C. O.; STRATONOVITCH, P.; STRECK, T.; SUPIT, I.; TAO, F.; THORBURN, P.; WAHA, K.; WALL, G. W.; WANG, E.; WHITE, J. W.; WOLF, J.; ZHAO, Z.; ZHU, Y. Similar estimates of temperature impacts on global wheat yield by three independent methods. Nature climate Change, v. 6, p. 1130 - 1137, 2016.

LOBELL, D. B.; BURKE, M. B.; TEBALDI, C.; MASTRANDREA, M. D.; FALCON, W. P.; NAYLOR, R. L. Prioritizing climate change adaptation needs for food security in 2030. Science, v. 319, p. $607-610,2008$

MAGRIN, G. O.; TRAVASSO, M. I.; RODRIGUEZ, G. R.; Climate change and wheat production in Argentina. International Journal of Global Warming, v. 1, n. 1-3, p. $214-226,2009$.

MARCELLOS, H.; SINGLE, W. V. The influence of cultivar, temperature and photoperiod on post-flowering development of wheat. Australian Journal of Agricultural Research, v. 23, p. 533 - 540, 1972.

MARENGO, J. A. Mudanças climáticas globais e seus efeitos sobre a biodiversidade: caracterização do clima atual e definição das alterações climáticas para o território brasileiro ao longo do século XXI. Brasília, DF: Ministério do Meio Ambiente, (2 ed.), 212p. (Biodiversidade, 26), 2007.

MARENGO J. A.; JONES R.; ALVES, L. M.; VALVERDE, M. C. Future change of temperature and precipitation extremes in South America as derived from the PRECIS regional climate modeling system. International Journal of Climatology, v. 29, p. 2241 - 2255, 2009.

MARENGO, J. A.; AMBRIZZI, T.; DA ROCHA, R. P.; ALVES, L. M.; CUADRA, S.V.; VALVERDE, M. C.; TORRES, R. R.; SANTOS, D.C.; FERRAZ, S. E. T Future change of climate in South America in the late twenty-first century: intercomparison of scenarios from three regional climate models. Climate Dynamics, v. 35, n. 6, p. 1073 - 1097, 2010.

MARENGO, J. A.; CAMARGO, C. C. Surface air temperature trends in Southern Brazil for 1960-2002. International Journal of Climatology, v. 28, p. 893 - 904, 2008

MASSIGNAM, A. M.; PANDOLFO, C.; RICCE, W. S.; SANTI, A.; MACHADO, L. N. Impacto das mudanças climáticas para o período futuro 2071-2100 no zoneamento do milho no Sul do Brasil. Agropecuária Catarinense, Florianópolis, v. 28, n. 2, p. 55 - 60, 2015.

MIRANDA, E. E. de; (Coord.). Brasil em Relevo. Campinas: Embrapa Monitoramento por Satélite, 2005. Disponível em: <http://www. relevobr.cnpm.embrapa.br>. Acesso em: 15 mar. 2010.

MOTA, F. S. Clima e zoneamento para a triticultura no Brasil. In: MOTA, F.S. (Ed.) Agrometeorologia do trigo no Brasil. Campinas: Sociedade Brasileira de Agrometeorologia, 1989. p. 5 - 35.

MYERS, S.; ZANOBETTI, A.; KLOOG, I.; HUYBERS, P.; LEAKEY, A. D. B.; BLOOM, A. J.; CARLISLE, E.; DIETTERICH, L. H.; FITZGERALD, G.; HASEGAWA, T.; HOLBROOK, N. M.; NELSON, R. L.; OTTMAN, M. J.; FABOY, V.; SAKAI, H.; SARTOR, K. A.; SCHWARTZ, J.; SENEWEERA, S.; TAUSZ, M.; USUI, Y. Increasing $\mathrm{CO}_{2}$ threatens human nutrition. Nature, v. 510, p.139 $-142,2014$

ORTIZ, R., SAYRE, K. D., GOVAERTS, B., GUPTA, R., SUBBARAO, G. V., BAN, T., HODSON, D., DIXON, J. M., ORTIZ-MONASTERIO, J. I., \& REYNOLDS, M. Climate change: Can wheat beat the heat? Agriculture, Ecosystems and Environment, v. 126, p. 46 - 58, 2008.

PANDOLFO, C.; PEREIRA, E. S.; RAMOS, A. M.; MASSIGNAM, A.; MIRANDA Jr., G. X.; THOMÉ, V. M. R. Sistema computacional para elaborar o zoneamento agrícola para o Estado de Santa Catarina - Zonexpert 1.0. In: CONGRESSO BRASILEIRO DE AGROMETEOROLOGIA, 11., 1999, Florianópolis, SC. Anais... Florianópolis, SC: Sociedade Brasileira de Agrometeorologia, 1999. 
PAULSEN, G. M. Wheat stand establishment. In: HEYNE, E.G. (ed.). Wheat and wheat improvement. 2nd ed. Agron. Monogr. 13. ASA, CSSA, and SSSA, Madison, WI. p. 384 - 415, 1987.

RAY, D. K.; MUELLER, N. D.; WEST, P. C.; FOLEY, J. A. Yield trends are Iinsufficient to double global crop production by 2050. PLoS ONE, v. 8, n. 6, p. $1-8,2013$.

RIBEIRO, T. L. P.; CUNHA, G. R.; PIRES, J. L. F.; PASINATO, A. Respostas fenológicas de cultivares brasileiras de trigo à vernalização e ao fotoperíodo. Pesquisa Agropecuária Brasileira, Brasília, v. 44, n. 11, p. $1383-1390,2009$.

SCHEEREN, P.L.; CUNHA, G.R.; QUADROS, F.J.S. de; MARTINS, L.F. Efeito do frio em trigo. Passo Fundo: Embrapa Trigo, 2000. 2p.html. 2 ilust. (Embrapa Trigo. Comunicado Técnico Online, 57). <Disponível: http:// www.cnpt.embrapa.br/biblio/p_co57.htm>. Acesso em: 14 mar. 2016.

SOLOMON, S.; PLATTNER, G. K.; KNUTTIC, R.; FRIEDLINGSTEIN, P. Irreversible climate change due to carbon dioxide emissions. PNAS, v. 106, p. 1704 - 1709, 2009.

STONE, P. J.; NICOLAS, M. E. Wheat cultivars vary widely in their responses of grain-yield and quality to short periods of post-anthesis heat-stress. Australian Journal of Plant Physiology, v. 21, p. 887 - 900, 1994.

STRECK, N. A.; ALBERTO, C. M. Estudo numérico do impacto da mudança climática sobre o rendimento de trigo, soja e milho. Pesquisa Agropecuária Brasileira, Brasília, v. 41, n. 9, p. 1351 - 1359, 2006.

THORNTHWAITE, C. W.; MATHER, J. R. The water balance. Publication of climatology, v. 8, 104 p., 1955.

TIAN, H.; LU, C.; CIAIS, P.; MICHALAK, A. M.; CANADELL, J. G.; SAIKAWA, E. HUNTZINGER, D. N.; GURNEY, K. R.; SITCH, S.; ZHANG, B.; YANG, J.; BOUSQUET, P.; BRUHWILER, L.; CHEN, G.; DLUGOKENCKY, E.; FRIEDLINGSTEIN, P.; MELILLO, J.; PAN, S.; POULTER, B.; PRINN, R.;
SAUNOIS, M.; SCHWALM, C. R.; WOFSY, S. C. The terrestrial biosphere as a net source of greenhouse gases to the atmosphere. Nature, v. 531, p. $225-228,2016$.

TRAVASSO, M. I.; MAGRIN, G. O.; BAETHGEN, W. E.; CASTAÑO, J. P.; RODRIGUEZ, G. R.; PIRES, J. L.; GIMENEZ, A.; CUNHA, G.; FERNANDES, M. Maize and soybean cultivation in Southeastern South America: adapting to climate change. In: LEARY, N.; ADEJUWON, J.; BARROS, V.; BURTON, I.; KULKARNI, J.; LASCO, R. (ed.). Climate change and adaptation. Sterling: Earthscan, 2008. p. 332 - 352.

TUBIELLO, F.N.; DONATELLI, M.; ROSENZWEIG, C.; STOCKLE, C.O. Effects of climate change and elevated $\mathrm{CO} 2$ on cropping systems: model predictions at two Italian locations. European Journal of Agronomy, v. 13, p. $179-189,2000$.

WALL, G. W.; GARCIA, R. L.; KIMBALL, B. A.; HUNSAKER, D. J.; PINTER Jr., P. J.; LONG, S.P.; OSBORNE, C. P.; HENDRIX, D. L.; WECHSUNG, F.; WECHSUNG, G.; LEAVITT, S. W.; LaMORTE, R. L.; IDSO, S. B. Interactive Effects of elevated carbon dioxide and drought on wheat. Agronomy Journal, v. 98, p. 354 - 381, 2006.

ZHENG, B.; CHENU, K.; CHAPMAN, S. C. Velocity of temperature and flowering time in wheat - assisting breeders to keep pace with climate change. Global Change Biology, v. 22, p. 921 - 933, 2016.

ZISKA, L. H.; MORRIS, C. F.; GOINS, E. W. Quantitative and qualitative evaluation of selected wheat varieties released since 1903 to increasing atmospheric carbon dioxide: can yield sensitivity to carbon dioxide be a factor in wheat performance? Global Change Biology, v. 10, p. 1810 $1819,2004$.

REFERENCIAÇÃO SANTI, A.; VICARI, M. B.; PANDOLFO, C.; DALMAGO, G. A.; MASSIGNAM, A. M.; PASINATO, A.

Impacto de cenários futuros de clima no zoneamento agroclimático do trigo na região Sul do Brasil. Agrometeoros, Passo Fundo, v.25, n.2, p.303-311, 2017. 


\title{
Future climate scenarios impact on wheat agroclimatic zoning in Southern Brazil
}

\author{
Anderson Santi ${ }^{1(*)}$, Matheus Boni Vicari ${ }^{2}$, Cristina Pandolfo ${ }^{3}$, Genei Antonio Dalmago ${ }^{1}$, Angelo Mendes Massignam ${ }^{3}$ and Aldemir \\ Pasinato $^{1}$ \\ ${ }^{1}$ Embrapa Trigo, Rodovia BR 285, km 294, Caixa Postal 3081, CEP 99050-970 Passo Fundo, RS, Brazil. E-mail: anderson.santi@embrapa.br, \\ genei.dalmago@embrapa.br and aldemir.pasinato@embrapa.br \\ ${ }^{2}$ University College London, Gower St, Bloomsbury, London WC1E 6BT, E-mail: matheus.boni.vicari@gmail.com \\ ${ }^{3}$ Empresa de Pesquisa Agropecuária e Extensão Rural de Santa Catarina - Epagri/CIRAM, Rodovia Admara Gonzaga, 1347, Itacorubi - Caixa Postal 502, \\ CEP 88034-901 Florianópolis, SC, Brazil. E-mails: cristina@epagri.sc.gov.br and massigna@epagri.sc.gov.br \\ ${ }^{(*)}$ Corresponding author
}

\section{ARTICLE INFO}

Article history:

Received 10 July 2017

Accepted 27 December 2017

\section{Index terms:}

air temperature

topothermal equations

sowing period

growing region

\section{ABSTRACT}

Climate change increases the risk on the global food supply by its significant effect on plants growth and development. Wheat has important role on human and animal nutrition and is highly responsive to climatic elements, and then vulnerable to climate change. This work aims to evaluate the impact of future climate scenarios on the agroclimatic zoning of wheat in Southern Brazil. There were considered two future climate scenarios, both established by the Intergovernmental Panel on Climate Change for the period from 2071 to 2100: A2, or more pessimistic and B2, or more optimistic. Both two scenarios were compared to the Current scenario (1961 - 1990). Wheat cultivation period indicated by agroclimatic zoning in all Southern Brazil will be reduced, with the temperature as the main reason for the reduction of the number of ten-day periods suitable for sowing. Wheat crop potential area will be reduced mainly in Paraná State in both scenarios and, in the west of Rio Grande do Sul State, only in the A2 scenario. 